\title{
OUTER COMPOSITIONS OF HYPERBOLIC/LOXODROMIC LINEAR FRACTIONAL TRANSFORMATIONS
}

\author{
JOHN GILL \\ Mathematics Department \\ University of Southern Colorado \\ Pueblo, CO 81001
}

(Received July 29, 1991)

ABSTRACT. It is shown, using classical means, that the outer composition of hyperbolic or loxodromic linear fractional transformations $\left\{f_{n}\right\}$, where $f_{n} \rightarrow f$, converges to $\alpha$, the attracting fixed point of $f$, for all complex numbers $z$, with one possible exception, $z_{o}$. I.e.,

$$
F_{n}(z):=f_{n} o f_{n-1} o \ldots o f_{1}(z) \rightarrow \alpha
$$

When $z_{o}$ exists, $F_{n}\left(z_{o}\right) \rightarrow \beta$, the repelling fixed point of $f$. Applications include the analytic theory of reverse continued fractions.

KEY WORDS AND PHRASES. Linear fractional transformations, continued fractions, fixed points. 1991 AMS SUBJECT CLASSIFICATION CODES. 30B70, 40A15, 40A30.

\section{INTRODUCTION.}

The iterative behavior of the non-singular linear fractional transformation $(L F T) f(z):=(a z+b) /(c z+d)$, $a, b, c, d \in C$ and $a d-b c \neq 0$, is well documented. For example, Ford [1] describes the "multiplier" form of $f(z)$ for LFT 8 that are loxodromic, hyperbolic, or elliptic:

$$
(f(z)-\alpha /(f(z)-\beta)=K(z-\alpha) /(z-\beta), K:=(a-c \alpha) /(a-c \beta) .
$$

Here $\alpha$ and $\beta$ are the two distinct fixed points of $f$. A special equation analogous to (1.1) exists for the one remaining type of $L F T$ - the parabolic transformation, having a single fixed point.

$K$ is called the multiplier of $f$ and provides an important means of classifying LFT's in terms of their fixed points. Briefly, (1.1) is either loxodromic or hyperbolic if $|K|<1$, and it is this combined case - representing "most" LFTs - we shall consider exclusively in this article. It is then easily seen from the multiplier form that $f^{n}(z) \rightarrow \alpha$ for all $z \neq \beta$, and $f^{n}(\beta) \equiv \beta$.

If $f(z):=a /(b+z), f^{n}(0)$ is the normal $n$th approximant of a periodic continued fraction

$$
\frac{a}{b}+\frac{a}{b}+\ldots
$$

Thus, this simple continued fraction converges to the attractive fixed point $\alpha$ of $f . \beta$ is called the repulsive or repellent fixed point of $f$. The modified continued fraction generated by $f^{n}(z)$ instead of $f^{n}(0)$ also converges to $\alpha$ for all $z \neq \beta$. For this special $f(z),|K|=|\alpha / \beta|$, so that $|\alpha|<|\beta|$.

Magnus and Mandell in 1971 [3] posed and answered the following question: If $\left\{f_{n}\right\}$ is a sequence of hyperbolic/loxodromic $(H / L) L F T$ s that converge to a $H / L L F T f(z)$, then what may one predict of the convergence behavior of the "inner" composition $F_{n}(z):=f_{1} o f_{2} o \ldots o f_{n}(z)$ for $z \in C$ ? They obtained the following result which resembles the iterative case.

THEOREM 1. If $\left\{f_{n}\right\}$ and $f=\operatorname{Lim} f_{n}$ are all $H / L$, then the inner compositional sequence $\left\{F_{n}(z)\right\}$ converges to a constant function for all values of $z \neq \beta$. 
As a consequence, the modified limit periodic continued fraction generated in this way by setting $f_{n}(z):=a_{n} /\left(b_{n}+z\right)$ with $f_{n}(z) \rightarrow f(z)=a /(b+z)$, and where all the $f_{n}$ 's and $f$ are $H / L$, converges to a constant for all $z \neq \beta$. This result has proven fruitful in both accelerating the convergence of such continued fractions and analytically continuing them beyond initial regions of convergence [4], [5].

In the current paper the question posed by Magnus and Mandell with regard to inner compositions is answered in the context of outer compositional structures:

$$
F_{n}(z):=f_{n} o f_{n-1} \ldots \ldots o f_{1}(z) \text {, where }\left\{f_{n}\right\} \text { and } f=\operatorname{Lim} f_{n} \text { are all } H / L .
$$

The proof of Theorem 1 is elementary, but complicated. Surprisingly, the proof of an analogous theorem for outer composition is, if anything, slightly more delicate. One would think that in $(1.2) F_{n}(z)$ becomes very like $f^{n}(z)$ for large values of $n$, and that this should simplify matters. However, the initial segment $F_{j}(z)$ for $j \ll n$ is not easy to control.

In analogy to Theorem 1 (and even closer to the simple iterative case) we shall prove

THEOREM 2. If $\left\{f_{n}\right\}$ and $f=\operatorname{Lim} f_{n}$ are all $H / L$, then the outer compositional sequence $\left\{F_{n}(z)\right\}$ described in (1.2) converges to $\alpha$, the attractive fixed point of $f$, for all values of $z$ except possibly one, $z_{o}$. In this exceptional case $F_{n}\left(z_{o}\right) \rightarrow \beta$, the repulsive fixed point of $f$.

As one example of Theorem 2, one easily obtains

COROLLARY 1. The modified reverse limit periodic continued fraction

$$
\frac{a_{n}}{b_{n}}+\frac{a_{n-1}}{b_{n-1}}+\ldots+\frac{a_{a}}{b_{a}+z}
$$

where $f_{n}(z):=a_{n} /\left(b_{n}+z\right)$ and $\operatorname{Lim} f_{n}(z)=f(z)=a /(b+z)$ are all $H / L$, converges to $\alpha$, the attractive fixed point of $f$ for all $z \in C$, with one possible exception.

In order to prove Theorem 2 , it is convenient to use a more general result from the analytic theory of contraction maps as applied to outer composition. The sufficiency part of the proof of the following theorem (all that is required in this exposition) is given in (1.2):

THEOREM 3. Let $\left\{g_{n}\right\}$ be a sequence of functions analytic on a simply connected region $S$ and continuous on the closure of $S$. Suppose there exists a compact set $\Omega$ contained in $S$ such that $\Omega \supset g_{n}(\Omega)$ for all $n$. Then, if $G_{n}(z):=g_{n} o \ldots o g_{1}(z), G_{n}(z) \rightarrow \alpha$, a constant, uniformly for all $z \in S$ if, and only if, the sequence $\left\{\alpha_{n}\right\}$ of fixed points of $\left\{g_{n}\right\}$ in $S$ converges to $\alpha$.

\section{PROOF OF THEOREM 2.}

An explicit expression for $f_{n}(z)$ from the multiplier form (1.1) is

$$
f_{n}(z)=\frac{\left(\alpha_{n}-K_{n} \beta_{n}\right) z+\alpha_{n} \beta_{n}\left(K_{n}-1\right)}{\left(1-K_{n}\right) z+K_{n} \alpha_{n}-\beta_{n}}
$$

Let us begin with a lemma that will prepare the way for the use of Theorem 3 in the present context. In all that follows it will be assumed that $K:=\operatorname{Lim} K_{n}, \alpha:=\operatorname{Lim} \alpha_{n}$, and $\beta:=\operatorname{Lim} \beta_{n}$ exist.

LEMMA 1. Let $R:=\rho|\alpha-\beta|, \rho:=(r-|K|) / r|1-K|,|K|<r<1$. For $n$ sufficiently large, there exists $t \in(r, 1)$ such that $|z-\alpha| \leq R \Rightarrow\left|f_{n}(z)-\alpha\right|<t R<R$.

PROOF. Writing

$$
\begin{gathered}
f_{n}(z)-\alpha=\left(f_{n}(z)-\alpha_{n}\right)+\varepsilon_{n} \quad\left(\varepsilon_{n}:=\alpha_{n}-\alpha\right) \\
=K_{n}\left(\alpha_{n}-\beta_{n}\right)\left[(z-\alpha)-\varepsilon_{n}\right] /\left[\left(1-K_{n}\right)(z-\alpha)+\alpha-\beta_{n}+K_{n} \varepsilon_{n}\right]+\varepsilon_{n}
\end{gathered}
$$

one gets

$$
\left|f_{n}(z)-\alpha\right| \leq\left|K_{n}\left(\alpha_{n}-\beta_{n}\right)\left(R+\left|\varepsilon_{n}\right|\right) /\left(\left|\alpha-\beta_{n}\right|-\left|K_{n} \varepsilon_{n}\right|-R\left|1-K_{n}\right|\right)+\right| \varepsilon_{n} \mid
$$

Letting $n \rightarrow \infty$ and replacing $R$ by its defined value, this last expression becomes: $r \rho|\alpha-\beta|=r R(<R)$.

Thus, for all $n$ sufficiently large and $|z-\alpha| \leq R$,

$$
\left|f_{n}(z)-\alpha\right|<t R<R, \text { for some } t \in(r, 1) \text {. }
$$


We next decompose the final segment of $F_{n+m}(z)$ in order to show that as $m$ increases applying $f_{n+m}$ is similar to applying $f$.

From (1.2) one can write

$$
f_{p}(z)=\lambda_{p}{ }^{-1} o K_{p} o \lambda_{p}(z), \text { where } \lambda_{p}(z):=\left(z-\alpha_{p}\right) /\left(z-\beta_{p}\right) \text { and } K_{p}(z):=K_{p} z
$$

Here, for all $p,\left|K_{p}\right| \leq K_{o}<1$.

Therefore

$$
\begin{aligned}
F_{n+m}(z)= & f_{n+m^{o}} \ldots o f_{n+1}\left(F_{n}(z)\right)= \\
& \lambda_{n+m}-1 o K_{n+m^{o}} \lambda_{n+m^{o}} \lambda_{n+m-1^{-1} o K_{n+m-1} \ldots o \lambda_{n+1}}{ }^{-1} o K_{n+1} o \lambda_{n+1}\left(z_{n}\right),
\end{aligned}
$$

where $z_{n}:=F_{n}(z)$. Then

$$
F_{n+m}(z)=\lambda_{n+m}{ }^{-1} o K_{n+m} o T_{m} o T_{m-1} o \ldots o T_{2}\left(w_{n}\right)
$$

where

$$
T_{p}(z):=\lambda_{n+p^{o} \lambda_{n+p-1}}{ }^{-1} \circ K_{n+p-1}(z) \text { and } w_{n}:=\lambda_{n+1}\left(F_{n}(z)\right) .
$$

The idea behind the proof of the next lemma is that $T_{p}(z) \approx K_{n+p-1}(z)$ for large values of $p$. This is a device initiated by Magnus and Mandell [3].

LEMMA 2. Suppose that $\left|w_{n}\right|<M(=M(z))$ for all $n$ sufficiently large. Then, for preassigned $\varepsilon>0$, there exists $N(=N(z))$ and $P(=P(z))$ such that $n>N$ and $p>P$ implies $\left|T_{p} o \ldots o T_{2}\left(w_{n}\right)\right|<2 \varepsilon /\left(1-K_{o}\right)$.

PROOF. From

$$
T_{2}\left(w_{n}\right)=\left\{K_{n+1} w_{n}\left(\beta_{n+1}-\alpha_{n+2}\right)+\left(\alpha_{n+2}-\alpha_{n+1}\right)\right] /\left[K_{n+1} w_{n}\left(\beta_{n+1}-\beta_{n+2}\right)+\left(\beta_{n+2}-\alpha_{n+1}\right)\right]
$$

one obtains

$$
\left|T_{2}\left(w_{n}\right)\right| \leq\left[K_{o} M\left|\beta_{n+1}-\alpha_{n+2}\right|+\left|\alpha_{n+2}-\alpha_{n+1}\right|\right] /\left[\left|\beta_{n+2}-\alpha_{n+1}\right|-K_{o} M\left|\beta_{n+1}-\beta_{n+2}\right|\right]<M
$$

and

$$
\left|T_{2}\left(w_{n}\right)-K_{n+1} w_{n}\right|<\varepsilon, \text { for large values of } n .
$$

Hence $\left|T_{2}\left(w_{n}\right)\right|<\varepsilon+\left|K_{n+1} w_{n}\right|<\varepsilon+K_{o} M$.

Similarly $\left|T_{3} o T_{2}\left(w_{n}\right)\right|<M$ and $\left|T_{3} o T_{2}\left(w_{n}\right)\right|<\varepsilon+K_{o}\left|T_{2}\left(w_{n}\right)\right|<\varepsilon\left(1+K_{o}\right)+K_{o} M^{2}$.

Continuing in this manner, one arrives at the general form $\left|T_{p} o . . o T_{2}\left(w_{n}\right)\right|<M$ and

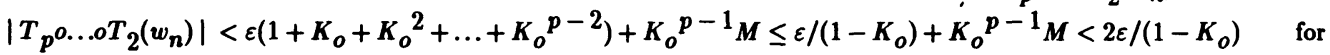
large $p$.

At several points later on we will refer back to this system of inequalities albeit under slightly different hypotheses.

In order to proceed, we need to know more about the exceptional point $z_{o}$ described in Theorem 2. If $f_{n} \equiv f$, then $z_{o}=\beta$. That $z_{o}$ can be any point in $C$ is easily seen by setting $f_{n} \equiv f$ for $n>1$ and allowing $f_{1}$ to be any $L F T$. Since $f_{1}$ is one to one in $C$, and $z_{o}=f_{1}^{-1}(\beta)$, clearly $z_{o}$ could be any point we wish, including $\alpha$.

It will become apparent later that the method of proof of Theorem 2 requires that $\left\{F_{n}(z)\right\}$ be uniformly bounded away from $\beta$ for large values of $n$. Consequently, the possibility that $\left\{F_{n}(z)\right\}$ has a cluster point at $\beta$ must be explored.

LEMMA 3. If $\left\{F_{n}(z)\right\}$ has a cluster point at $\beta$, then $F_{n}(z) \rightarrow \beta$.

PROOF. Suppose that $\left\{F_{n}(z)\right\}$ has a cluster point at $\beta$, but does not converge to $\beta$. Then there exists an additional cluster point $\beta^{*} \neq \beta$. Assum $\beta^{*} \neq \infty$ (a slight variation on the following argument works for this specia case). 
No matter how large $N$ is, there exists $n>N$ such that $F_{n}(z) \in N_{\varepsilon}\left(\beta^{*}\right)$, where $\varepsilon>0$ is chosen to exclude by a large margin $\beta$ or any $\beta_{j}$ from $N_{\varepsilon}\left(\beta^{*}\right)$. We will show that this has the effect of eliminating the possibility of $\beta$ being a cluster point of the sequence, thus providing a contradiction.

Under these conditions $\left|\lambda_{n+1}\left(F_{n}(z)\right)\right|<M$ for an infinite subsequence of $n$ 's. For such an $n$ large enough the entire structure of the proof of Lemma 2 remains intact, thus giving, for a suitable choice of $\varepsilon$, $\left|T_{p} o \ldots T_{2}\left(w_{n}\right)\right|<2 \varepsilon /\left(1-K_{o}\right)=1$ for all $p$ sufficiently large.

$$
\begin{aligned}
& \text { Now }\left|F_{n+p}(z)-\beta_{n+p}\right|=\left|\lambda_{n+p}{ }^{-1} o K_{n+p}\left(T_{p} \ldots \ldots o T_{2}\left(w_{n}\right)\right)-\beta_{n+p}\right| \\
& \geq\left|\beta_{n+p}-\alpha_{n+p}\right| /\left(1+\left|K_{n+p}\right|\left|T_{p} o . . o T_{2}\left(w_{n}\right)\right|\right) \\
& >|\beta-\alpha| /(2(1+|K|)) \text { for all } p \text { sufficiently large. }
\end{aligned}
$$

Therefore, it is not possible that $\left\{F_{j}(z)\right\}$ has a cluster point at $\beta$. ( $\left.\rightarrow \leftarrow\right)$

Next, we see that $z_{o}$, if it exists, is unique.

LEMMA 4. There exists no more than one value $z_{o}$ such that $F_{n}\left(z_{o}\right) \rightarrow \beta$.

PROOF. Suppose there are two such values, $z_{1}$ and $z_{2}$. Set $V_{n}:=F_{n}\left(z_{1}\right)$ and $W_{n}:=F_{n}\left(z_{2}\right)$. Observe that $V_{n} \neq W_{n}$ since $F_{n}$ is one to one. For large $n$ (using a local uniform convergence argument) $\left|f_{n+1}\left(V_{n}\right)-f_{n+1}\left(W_{n}\right)\right| /\left|V_{n}-W_{n}\right| \approx\left|f_{n+1}\left(V_{n}\right)\right| \approx f^{\prime}(\beta) \mid>1$.

Therefore, $\left|V_{n+1}-W_{n+1}\right| /\left|V_{n}-W_{n}\right|$ for all $n$ sufficiently large. Hence, one of $\left\{V_{n}\right\}$ or $\left\{W_{n}\right\}$ does not converge to $\beta$. $(\rightarrow \leftarrow)$

QED

It is now possible to complete the proof of Theorem 2.

If $z \neq z_{0}$, then Lemmas 3 and 4 tell us that there exists $D>0$ such that $\left|F_{n}(z)-\beta_{n+1}\right|>D$ for all $n$ sufficiently large. We use this to insure the boundedness of $\left\{F_{n}(z)\right\}$ for large $n$. Then it is possible to show that $\left|F_{n}(z)-\alpha\right|<\rho|\alpha-\beta|$ for large $n$, thus allowing the use of Lemma 1 and Theorem 3.

Suppose that $\left\{F_{n}(z)\right\}$ has a cluster point at $\infty$. Choose $n$ large enough to guarantee that $\left|\lambda_{n+1}\left(F_{n}(z)\right)\right| \approx 1$ and that the inequalities of Lemma 2 are valid. Then, for suitable $\varepsilon>0,\left|T_{p^{o} \ldots o T_{2}}\left(w_{n}\right)\right|<\rho / 4 K_{o}<1 / 2 K_{o}$.

It then follows that

$$
\begin{aligned}
& \left|F_{n+p}(z)\right|=\left|\lambda_{n+p} p^{-1} o K_{n+p}\left(T_{p} o . . o T_{2}\left(w_{n}\right)\right)\right| \\
& \leq\left(\left|\beta_{n+p} K_{n+p}\left(T_{p} o \ldots o T_{2}\left(w_{n}\right)\right)\right|+\left|\alpha_{n+p}\right|\right) /\left(1-\left|K_{n+p}\left(T_{p} \ldots \ldots o T_{2}\left(w_{n}\right)\right)\right|\right) \\
& \leq\left|\beta_{n+p}\right|+2\left|\alpha_{n+p}\right|<B \text { for all } p \text { sufficiently large. }(\rightarrow \leftarrow)
\end{aligned}
$$

Therefore, for all sufficiently large $n,\left|\lambda_{n+1}\left(F_{n}(z)\right)\right|<M$.

Lemma 2 then insures that $\left|T_{p} o \ldots o T_{2}\left(w_{n}\right)\right|<\rho / 4 K_{o}<1 / 2 K_{o}$, for all sufficiently large values of $p$. Next,

$$
\begin{aligned}
& \left|F_{n+p}(z)-\alpha\right| \leq\left|F_{n+p}(z)-\alpha_{n+p}\right|+\left|\varepsilon_{n+p}\right| \quad \text { (recall: } \varepsilon_{n}:=\alpha_{n}-\alpha \text { ) }
\end{aligned}
$$

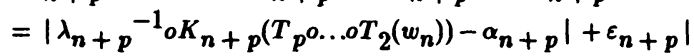

$$
\begin{aligned}
& \leq K_{o}\left|T_{p} o \ldots o T_{2}\left(w_{n}\right)\right|\left|\beta_{n+p}-\alpha_{n+p}\right| /\left(1-K_{o}\left|T_{p^{o} \ldots o T_{2}}\left(w_{n}\right)\right|\right)+\left|\varepsilon_{n+p}\right| \\
& <(\rho / 2)\left|\beta_{n+p}-\alpha_{n+p}\right|+\left|\varepsilon_{n+p}\right|<\rho|\beta-\alpha| \text { for all } p \text { sufficiently large. }
\end{aligned}
$$

Therefore $z_{p}:=F_{n}+p(z)$ lies in the disk $(|z-\alpha| \leq R)$ of Lemma 1 .

Theorem 3 then implies $\operatorname{Lim}_{m \rightarrow \infty} f_{n+m^{o}} f_{n+m-1}$ o..of $f_{n+p+1}\left(z_{p}\right)=\alpha$.

Hence $\operatorname{Lim}_{n \rightarrow \infty} F_{n}(z)=\alpha$.

\section{REFERENCES}

1. FORD, L. Automorphic Functions, 2nd Ed., Chelsea, New York, 1951.

2. GILL, J. The Use of the Sequence $F_{n}(z)=f_{n} o \ldots o f_{1}(z)$ in Computing Fixed points of Continued Fractions, Products, and Series, J. App. Num. Math., 8 (1991), 469-476.

3. MAGNUS, A. and MANDELL, M. On Convergence of Sequences of Linear Fracitonal Transformations, Math. Z. 115 (1970), 11-17.

4. THRON, W. and WAADELAND, H. Accelerating Convergence of Limit Periodic Continued Fractions $K\left(a_{n} / 1\right)$, Numer. Math. 34 (1980), 155-170.

5. THRON, W. and WAADELAND, H. Analytic Continuation of Functions Defined by Continued Fractions, Math. Scand. 47 (1980), 72-90. 


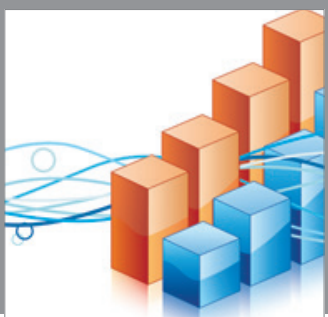

Advances in

Operations Research

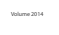

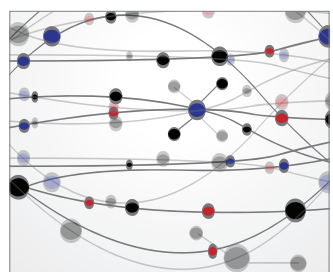

\section{The Scientific} World Journal
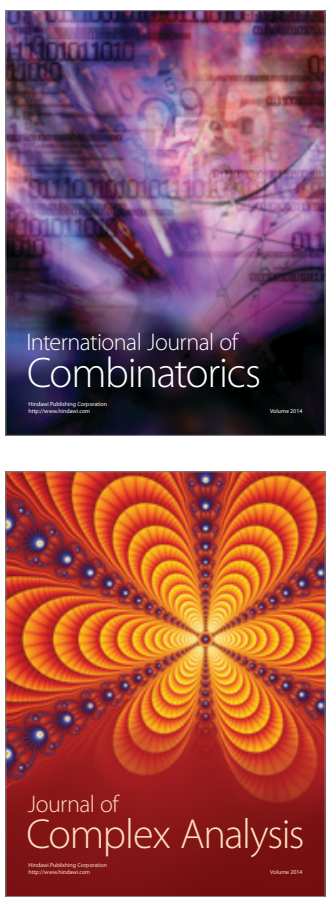

International Journal of

Mathematics and

Mathematical

Sciences
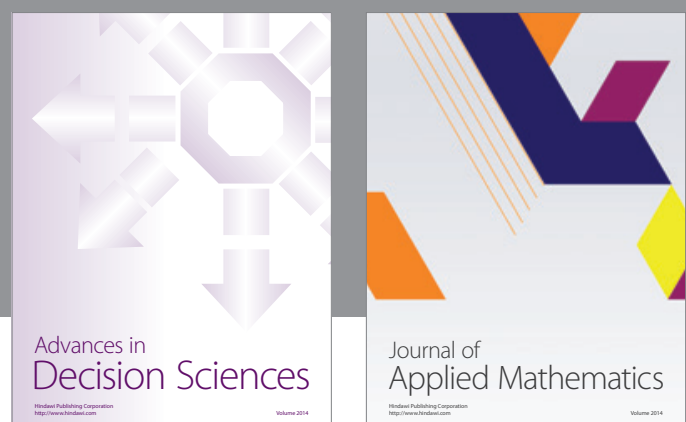

Journal of

Applied Mathematics
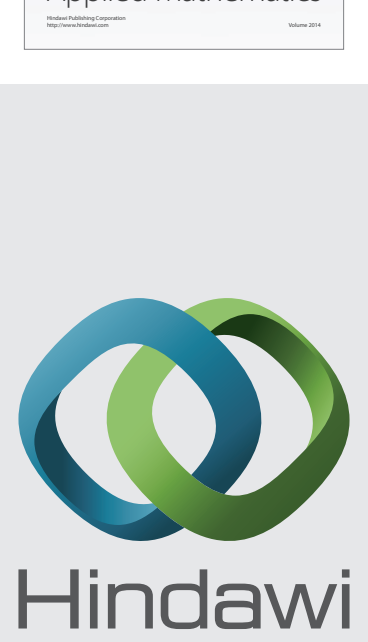

Submit your manuscripts at http://www.hindawi.com
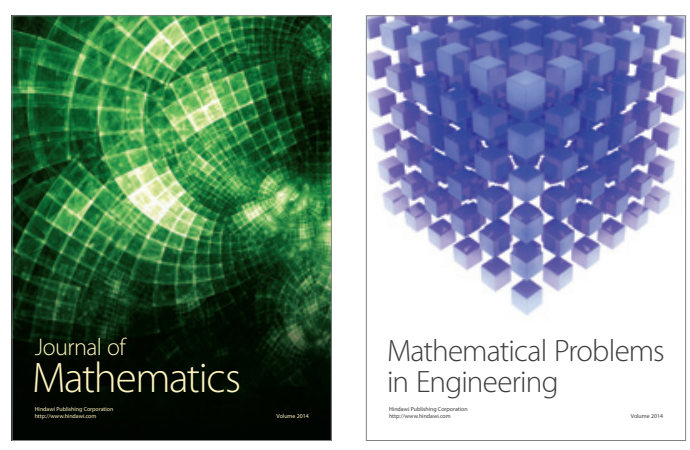

Mathematical Problems in Engineering
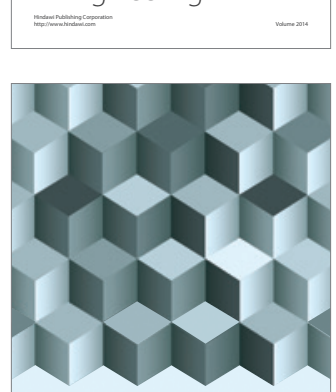

Journal of

Function Spaces
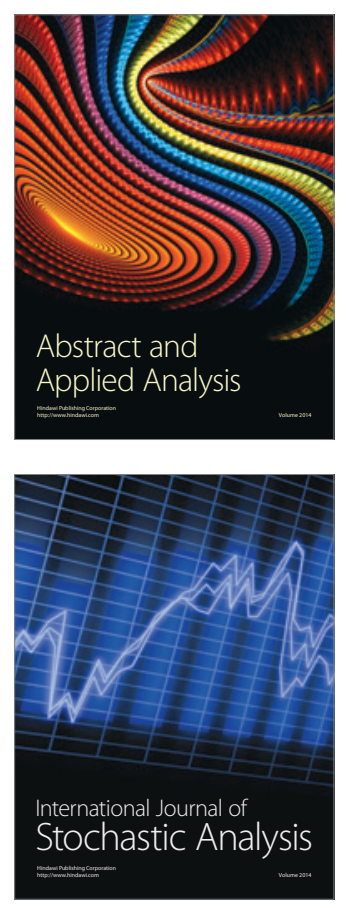

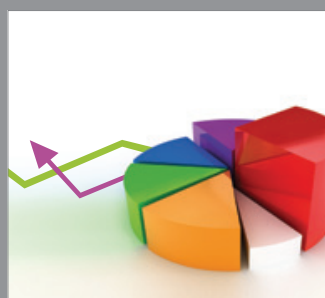

ournal of

Probability and Statistics

Promensencen
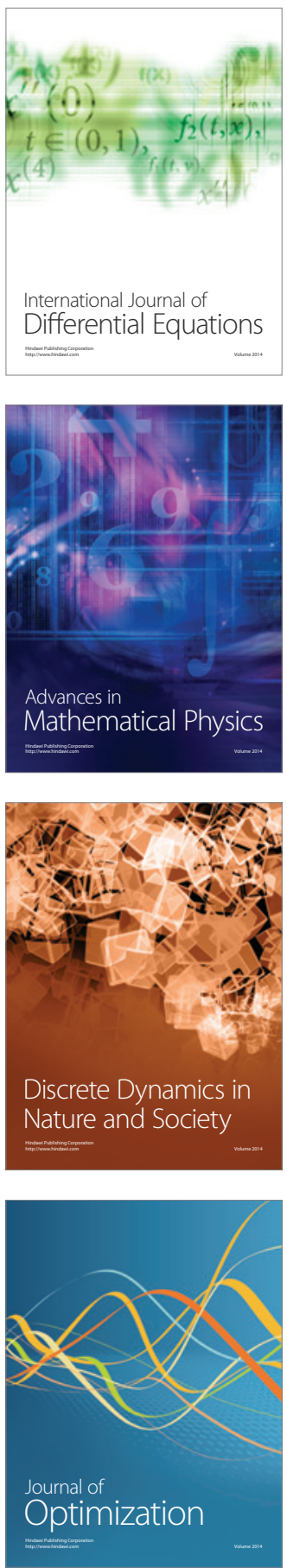